\title{
Arylsulfonyl Derivatives of 11-Azaartemisinin: Approaching New Polymorphs via Seeds of Molecular Analogues
}

\author{
Madiha Nisar, Herman H-Y. Sung and Ian D. Williams* \\ Department of Chemistry, Hong Kong University of Science and Technology, \\ Clear Water Bay, Hong Kong, China. E-mail: $\underline{\text { mnisar@connect.ust.hk }}$
}

11-Azaartemisinin and its derivatives are receiving renewed attention ${ }^{1,2}$ since they are active against drugresistant malarial parasites. Among the active derivatives are N-sulfonyl compounds. Those bearing an aryl group may be readily varied by changing substituents on the Ph ring. Even with small changes (e.g. 2-, 3- or 4- F, Cl, Br, I, Me) many of these compounds do not make isostructural phases with either the parent $\mathrm{Ph}$ compound or each other, despite only minor change in molecular formula.

In this paper we present our study of what may be termed 'quasi-polymorphs' in this series of compounds, explaining how and why their structures are different. We also report on attempts to use hetero-crystal seeding to grow real polymorphs for some of these compounds. These principles could be applied as an approach to otherwise inaccessible solid state forms of many drugs, or indeed organic compounds in general.
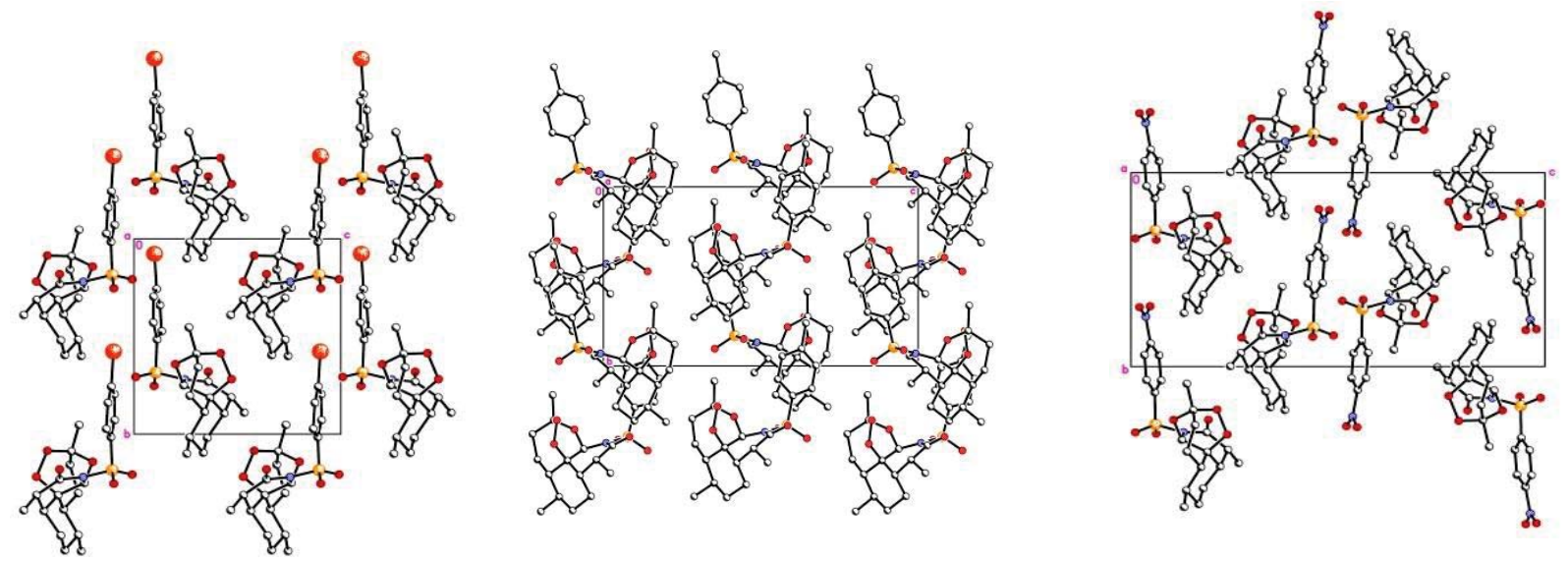

We are grateful to the Research Grants Council of Hong Kong for funding of this work (grant 16306515).

[1] Harmse, R.,Coertzen, D., Wong, H. N., Smit, F. J., van der Watt, M. E., Reader, J., Nondaba, S. H., Birkholz, L-M., Haynes, R. K.\& N’Da, D. ChemMedChem 2017, 12, 2086-2093.

[2] Nisar, M.; Sung, H.H-Y.; Puschmann, H.; Lakerveld, R.; Haynes, R.K.; Williams, I.D. CrystEng

Comm, 2018, 20, 1205-1219 